\title{
Inferring context-sensitive Probabilistic Boolean Networks from gene expression data under multi-biological conditions
} Le $\mathrm{Yu}^{*}$ and Stephen Marshall

\author{
Address: Department of Electronic and Electrical Engineering, University of Strathclyde, Royal College Building, Glasgow, G1 1XW, UK \\ Email: Le Yu* - L.Yu@eee.strath.ac.uk \\ * Corresponding author
}

from BioSysBio 2007: Systems Biology, Bioinformatics and Synthetic Biology

Manchester, UK. II-13 January 2007

Published: 8 May 2007

BMC Systems Biology 2007, I(SuppI I):P63 doi: I0.II86/I752-0509-I-SI-P63

This abstract is available from: http://www.biomedcentral.com/I752-0509/I?issue=SI

(c) 2007 Yu and Marshall; licensee BioMed Central Ltd.

\section{Background}

In recent years biological microarrays have emerged as a high-throughput data acquisition technology in bioinformatics. In conjunction with this, there is an increasing need to develop frameworks for the formal analysis of biological pathways. A modeling approach defined as Probabilistic Boolean Networks (PBNs) was proposed for inferring genetic regulatory networks [1]. This technology, an extension of Boolean Networks [2], is able to capture the time-varying dependencies with deterministic probabilities for a series of sets of predictor functions.

\section{Methods and materials}

This report addresses a new modeling approach for analyzing temporal data sequences of gene expression using Probabilistic Boolean Networks. The modeling is applied in the context of interferon pathway biology to the analysis of gene interaction networks. Based on the analysis of gene expression measurements of macrophage cells challenged with virus infection and interferon treatment (see Figure 1), we demonstrate that switch-like phenomena exists. The switch like responses are particularly amenable to probabilistic modeling and accordingly, we develop a new model extending the Probabilistic Boolean Networks (PBNs) concept for the inference of gene regulatory networks from gene expression time-course data under different biological conditions. The model is a collection of traditional Probabilistic Boolean Networks. We further investigate and analyse the length of the attractor basin in the Boolean Networks inferred from the data under the requirement of contextual data consistency [3], and identify the network selection probabilities of the model according to the frequencey distribution of the observed gene expression data.

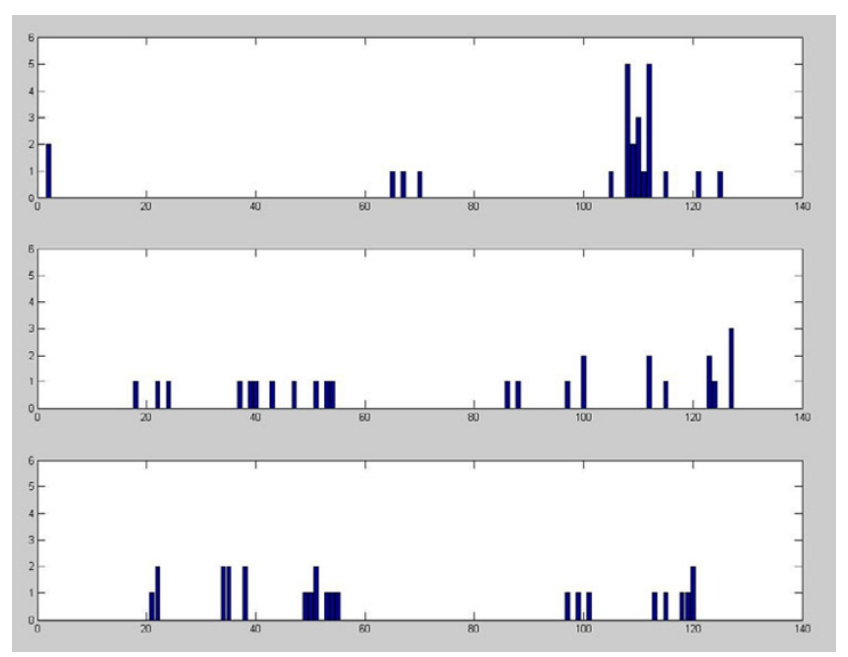

\section{Figure I}

The steady states distribution the genes varying under the 3 different biological conditions: interferon treatment only (INFg), viral infection with interferon treatment (C3X_INFg) and viral infection only (C3X). 
Table I: Attractors and probabilities for the context in the network of macrophage cells pathway varying under the biological conditions of interferon treatment only (INFg).

\begin{tabular}{|c|c|c|}
\hline Context I & $1,64,69,107,108,109,114,120$ & $3.75 \%$ \\
\hline Context 2 & $1,64,69,107,108,111,114,120$ & $6.25 \%$ \\
\hline Context 3 & $1,64,69,107,109,114,120$ & $5.625 \%$ \\
\hline Context 4 & $1,64,69,107,109,111,114,120$ & $9.375 \%$ \\
\hline Context 5 & $1,64,69,111,108,109,114,120$ & $3.75 \%$ \\
\hline Context 6 & $1,64,69,111,108,114,120$ & $6.25 \%$ \\
\hline Context 7 & $1,64,69,111,109,111,114,120$ & $15 \%$ \\
\hline Context 8 & $1,66,69,107,108,109,114,120$ & $3.75 \%$ \\
\hline Context 9 & $1,66,69,107,108,111,114,120$ & $6.25 \%$ \\
\hline Context 10 & $1,66,69,107,109,114,120$ & $5.625 \%$ \\
\hline Context II & $1,66,69,107,109,111,114,120$ & $9.375 \%$ \\
\hline Context 12 & $1,66,69,111,108,109,114,120$ & $3.75 \%$ \\
\hline Context 13 & $1,66,69,111,108,114,120$ & $6.25 \%$ \\
\hline Context 14 & $1,66,69,111,109,111,114,120$ & $15 \%$ \\
\hline
\end{tabular}

\section{Results}

Table 1 shows attractors for the network of macrophage cells pathway varying under the biological conditions of interferon treatment only (INFg). The network selection probabilities display different values for the different context. In this model, the structure of the constitute Boolean networks remains stable, whereas the network selection probabilities change in response to the different biological context. This is consistent with a fixed finite discrete state space but different stationary distributions. This model keeps the rule-based structure while allowing for uncertainty. It is suitable for the inference of the gene regulatory network from gene expression data in different biological conditions.

\section{Conclusion}

We applied a novel approach to model the gene regulator activities under different biological conditions. A method for identifying the model parameters from real time course gene expression profiles has been successfully conducted.

\section{References}

I. Shmulevich I, Dougherty ER, Kim S, Zhang W: Probabilistic Boolean Networks: A Rule-Based Uncertainty Model for Gene Regulatory Networks. Bioinformatics 2002, 18:26I-274.

2. Kauffman SA: The Origins of Order: Self-organization and Selection in Evolution. New York: Oxford University Press; 1993.

3. Dougherty ER, Xiao Y: Design of Probabilistic Boolean Networks under the Requirement of Contextual Data Consistency. IEEE Trans on Signal Processing 2006, 54(9):3603-36I3.

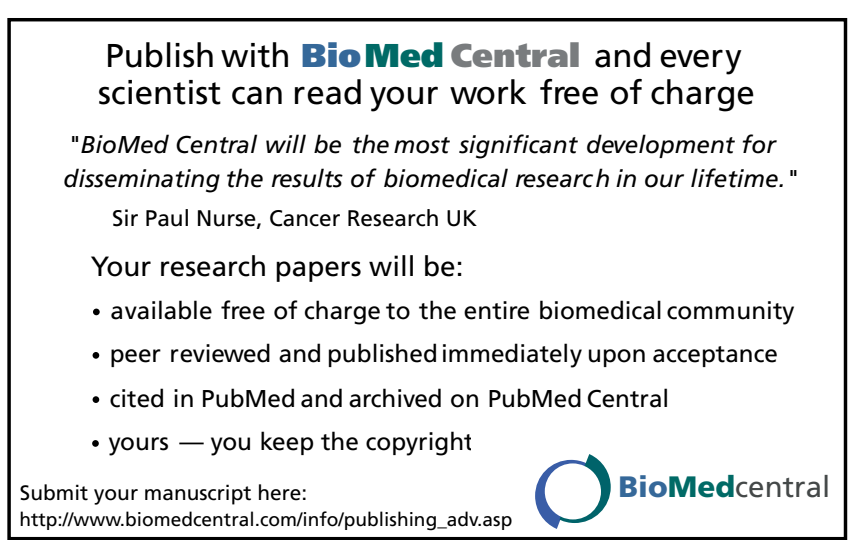

